\title{
Coulisses
}

Revue de théâtre

7| Printemps 1993

Varia

\section{Brève exploration lexicale aux sources du fleuve Scène}

Jean-Paul Colin

\section{OpenEdition}

1 Journals

Édition électronique

URL : http://journals.openedition.org/coulisses/2106

DOI : $10.4000 /$ coulisses. 2106

ISSN : 2546-9460

Éditeur

Presses universitaires de Franche-Comté

\section{Édition imprimée}

Date de publication : 1 avril 1993

Pagination : 4-6

ISSN : 1150-594X

\section{Référence électronique}

Jean-Paul Colin, «Brève exploration lexicale aux sources du fleuve Scène », Coulisses [En ligne], 7

Printemps 1993, mis en ligne le 15 mars 2019, consulté le 24 octobre 2019. URL : http://

journals.openedition.org/coulisses/2106 ; DOI : 10.4000/coulisses.2106

Ce document a été généré automatiquement le 24 octobre 2019.

Coulisses 


\title{
Brève exploration lexicale aux sources du fleuve Scène
}

\author{
Jean-Paul Colin
}

1 Le théâtre est vieux comme le monde des hommes et des femmes, et l'on imagine volontiers un dialogue préhistorique du genre: «Tu vas pas encore me faire une scène ? - Si, ma vieille, et je vais même te mettre en pièce(s)... » Ainsi commence la tragédie.

2 Nous aurions du mal à remonter au-delà des anciens Grecs, orfèvres, comme chacun

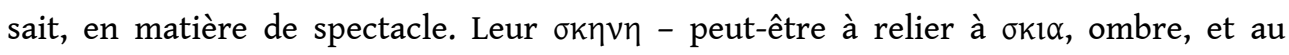
sanskrit châya, idem - avait le sens global de « construction légère servant d'abri », ce qui permettait de désigner tantôt une cabane, une hutte, tantôt une tente (notamment militaire; on appelait Arabes scénites [Académie, 1844] les peuplades nomades qui vivaient sous la tente), tantôt une boutique. Et aussi, bien entendu, une construction en bois et couverte où l'on jouait des pièces de théâtre; plus précisément, il s'agissait, dans cette construction, du lieu où jouaient les acteurs, tandis que le chœur antique évoluait sur la $\Theta v \mu \varepsilon \lambda \eta$, sorte d'estrade. Aristophane, dans La Paix et Platon, dans son

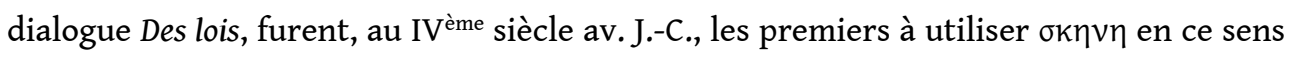
culturel. Dès la langue grecque, le mot en vient aussi à signifier « fiction de théâtre » et " mensonge ». Les Latins ne feront qu'emprunter le substantif très directement, sous la forme scena ou scaena. en lui donnant le sens de «scène d'un théâtre, théâtre, scène publique ».

3 Les représentations des Mistères médiévaux ignoraient totalement la notion de scène, puisqu'elles se déroulaient à ciel ouvert, souvent sur le parvis (ou paradis) des églises. C'est en 1375 seulement que scène fait son apparition en français, de même que

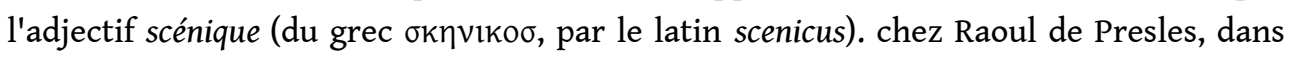
l'expression: faiseurs de jeux scéniques, et uniquement au sens de «représentation théâtrale de l'Antiquité ». A la fin du XVI ${ }^{e ̀ m e}$ siècle, les choses se précisent, le mot prenant le sens concret de "partie du théatre généralement garnie d'un décor, dans laquelle jouent les acteurs». 
Mais on ne sera pas surpris que la majorité des acceptions, propres et figurées, de scène proviennent $\mathrm{du}$ XVII ème siècle, qui fut véritablement celui de l'invention du théâtre moderne. Curieusement, c'est Crespin, dès 1637, qui crée l'expression scène-à-faire, se rapportant déjà plus à l'écriture qu'au jeu lui-même. En 1660, le cardinal de Retz emploie le mot au sens de "spectacle en général», et Corneille crée en 1642 le syntagme verbal mettre sur la scène au figuré, dans l'Examen de Polyeucte. Pour Boileau, en 1669, c'est $1^{\prime}$ «action théâtrale »: on rencontre dans ses Épitres VII et VIII les syntagmes scène tragique au sens $d^{\prime}$ ' art de la tragédie» et scène française au sens d'« ensemble des pièces de théâtre en français », et pour Molière, en 1671, 1'« ensemble des décors ». En 1675, la scène désigne 1'« art dramatique» (chez Boileau encore). Furetière, dans son fameux dictionnaire (1690), donne la définition classique de " théâtre » au sens large et «lieu où se passe l'action qu'on représente ». L'Académie française ajoute en 1694 le tour paraitre sur la scène: l'essentiel du champ lexicosémantique est en place. Curieusement, il faudra attendre 1738 pour découvrir le sens technique ancien de "mur de fond ». En 1776, selon P. Enckell, la scène lyrique désigne l'Opéra (de Paris, bien sûr...). Le tour entrer en scène, qui nous paraît si naturel, n'est enregistré qu'en 1835, dans le Dictionnaire de l'Académie française, et il faut attendre le début du XX⿳亠㐅⿵冂⿰⿱丶㇀⿱㇒丶⿱一土⿵冂丶 scène, l'expression est rare et peu lexicalisée, et le tour substantival se limite généralement (à la différence d'entrée en scène) à sortie (ou fausse sortie!) Le jeu de scène ne figure qu'en 1873, chez Larousse, au sens d'«effet produit par les gestes ou la mimique de l'acteur ", mais on le rencontre plus tard avec le sens $\mathrm{d}^{\prime}$ « indication fournie par l'auteur dans son texte à l'intention des acteurs ». Avec faire une scène à quelqu'un (1782, dans Adèle et Théodore, de Mme de Genlis), on est en pleine métaphore, le théâtre descend dans la rue et dans la maison de chacun; on aura scène de ménage en 1876 (Larousse) et le populaire jouer la grande scène du II en 1882 dans un roman des Goncourt, La Faustin. Mais dès le Grand siècle, le père jésuite Bouhours écrivait, dans ses Remarques nouvelles sur la langue françoise (1675): « Ce mot [i.e. scène] est depuis quelques années fort à la mode dans un certain tour: Ce fut une étrange scène, il a donné une plaisante scène au public. Ce mot est venu de la Cour ».

Balzac, en 1830, généralisera également ce mot dans le sens non-théâtral d'« action partielle ayant une unité ", fameusement illustré dans les Scènes de la vie de Bohème de Murger, en 1848. Enfin, le méconnu Complément du Dictionnaire de l'Académie française fournit en 1844 les emplois techniques et quelque peu pédants de scène ductile pour « décor coulissant longitudinalement » et de scène versatile pour « décor triangulaire et pivotant permettant de passer quasi instantanément du tragique au comique ou au satirique ». La scène peut également, bien entendu, être qualifiée " physiquement » de fixe, coulissante, tournante, etc.

6 En ce qui concerne les dérivés, on a vu que le plus ancien, scénique, est ancien, et contemporain des premières apparitions de scène en français. En dehors de scénographie , né en 1545 dans une traduction de Vitruve au sens non-théâtral d'« art ou action de

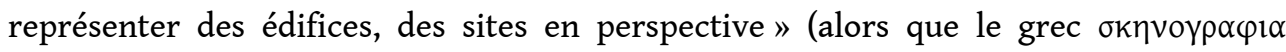
désignait déjà un "décor de peinture pour le théâtre ») et qui ne revêt l'acception moderne d'« art et science de l'agencement et du matériel de scène » qu'en... 1943, les autres mots suffixés à partir de scène sont beaucoup plus tardifs, à commencer par

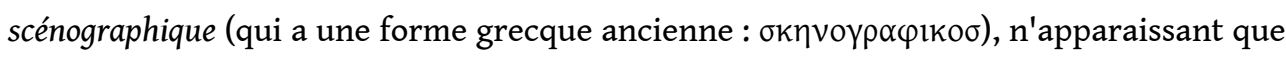

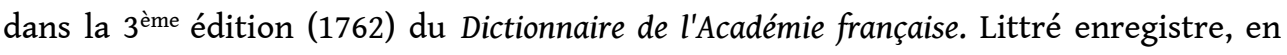


1873, scénographiquement, "selon les règles de la scénographie; en perspective ". Stendhal, audacieux néologue, a fabriqué le 26 janvier 1806, dans son célèbre Journal, le verbe scénifier, "diviser une pièce en scènes ", qui n'eut point de lendemain... (d'après J. Suchy, dans le $n^{\circ} 30$ des Datations et documents lexicographiques).

$7 \quad$ L'adjectif scénique ne s'est développé dans l'adverbe scéniquement qu'en 1876 (chez A. Daudet, cité par Littré), et le scénographe, limité en 1829 au sens d'« artiste fabriquant des décors en perspective ", n'a pris le sens actuel qu'en 1964, dans le Grand Larousse encyclopédique.

Enfin, le néologisme scénologie désigne, à partir de 1964 (Grand Larousse encyclopédique) la « science de la mise en scène au théâtre ».

9 Une curiosité historique et orthographique : alors que le diminutif de pièce est piécette (mais guère, il est vrai, en parlant de théâtre), celui de scène semble être... saynète, de l'espagnol sainete, qui désigne une courte pièce bouffonne (et n'a absolument aucun rapport avec le latin scaena). Le mot apparaît en France en 1764, et donne lieu à une très fréquente faute d'orthographe.

On remarquera que scène et ses dérivés n'ont pas débordé dans la cinématographie, où c'est la séquence qui joue plus ou moins le rôle d'unité narrative combinable... Quant au scénario, nous n'en dirons rien, il appartient à une tout autre famille que le mot scène, en passant par l'italien...

11 Comme partie d'un acte, le mot scène entre dans des syntagmes nominaux, qui apportent des précisions d'ordre textuel ou littéraire à notre domaine : la scène muette (1735, Marivaux) est évidemment (?) et paradoxalement celle dans laquelle les personnages ont un jeu muet.

Quant à la scène primitive (1954, traduction par Marie Bonaparte de la fameuse Urszene inventée en 1897, dans L'Homme aux loups, par S. Freud) ou originaire (1968, Supplément au Grand Larousse Encyclopédique en 10 volumes), elle ne fait partie que du théâtre intérieur de nos fantasmes, et a le sens d'«expérience traumatique observée ou imaginée par l'enfant concernant une scène de rapports sexuels entre les parents ».

13 Les locutions comprenant le mot scène sont assez nombreuses : nous venons d'en voir quelques-unes. La mise en scène date de 1800, d'après B. Quémada, ce qui laisse supposer que le tour mettre en scène est au moins contemporain, sinon antérieur, bien qu'on ne le trouve attesté qu'en 1835 dans le Dictionnaire de l'Académie. Le metteur en scène fait son apparition tardivement, en 1859, dans un aimable roman de J.-P. Stahl (pseudonyme de l'éditeur Hetzel), Histoire d'un homme enrhumé. Ouvrir la scène, au sens d'« apparaître le premier sur le théâtre ", qu'on trouve aussi dans le Dictionnaire de l'Académie en 1835 (5 ème édition), est aujourd'hui désuet. Porter à la scène n'est employé qu'en 1899 pour la première fois, par P. Lalo. Quitter la scène, au sens de « prendre sa retraite, en parlant d'un acteur ", est du XX ${ }^{\text {ème }}$ siècle seulement. Enfin, l'opposition qui parait si classique : à la scène comme à la ville, surgit chez Théophile Gautier, en 1874, dans ses Portraits contemporains... Les mots préfixés sont extrêmement rares : l'antique proscenium, du

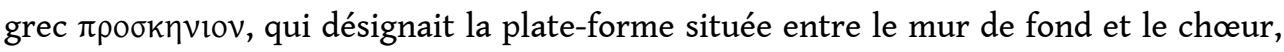
ne figure qu'en 1762 dans le Dictionnaire de l'Académie. Paul Claudel, non-conformiste, l'emploie en 1929 comme synonyme d'avant-scène, dans Le Livre de Christophe Colomb. Quant à ce dernier mot, il apparaît en 1570, d'abord comme synonyme... de proscenium, puis désigne, à partir de 1790, l'espace compris entre la rampe et le premier plan du décor. Enfin, le sens moderne de «loge située sur les côtés et tout à l'avant de la 
scène ", date de 1835. Le devant de la scène, qu'on rencontre en 1882 dans Les Corbeaux d'Henri Becque, s'emploie aujourd'hui davantage au figuré...

Quittons enfin, quelque peu, le registre «noble » : le peuple va aussi au théâtre, mais ne s'y amuse pas toujours : si l'on s'ennuie au poulailler, on peut néanmoins se rincer l'œil en « zyeutant » dans les avant-scènes, c'est-à-dire, pour les titis parisiens contemporains du Dictionnaire de la langue verte d'Alfred Delvau (1867), en contemplant ce que laissent déborder de leur corsage les opulentes spectatrices du balcon situé juste au-dessous d'eux ( $d$ 'où la variante bien connue : $Y$ a du monde au balcon) !

15 Après un tel tour d'horizon, comment terminer honorablement ? En cachant, bien sûr, ce magique saint des saints, je veux dire la scène, au cri populaire et performatif de : «Rideau !» et en tournant les talons quand il en est temps encore, avant d'être atteint, comme les acteurs ringards, par la... scénilité (astuce relevée dans le Nouvel Observateur $\mathrm{du} 1^{\mathrm{er}}$ août 1986, par le lexicologue anglais K.E. George).

Plaudite, cives !

\section{AUTEUR}

\section{JEAN-PAUL COLIN}

Professeur de linguistique à l'Université de Franche-Comté, il est l'auteur de plusieurs

dictionnaires très particuliers. 